\title{
Abnormal polarization of macrophage-like cells in the peripheral blood of patients with glioma
}

\author{
YAWEI GUO $^{1 *}$, WENMING HONG ${ }^{1,2^{*}}$, PENGYING ZHANG ${ }^{1}$, DAFEI HAN ${ }^{1}$, \\ YILONG FANG $^{1}$, JIAJIE TU ${ }^{1}$ and WEI WEI ${ }^{1}$
}

\begin{abstract}
${ }^{1}$ Key Laboratory of Anti-Inflammatory and Immune Medicine, Ministry of Education, Anhui Collaborative Innovation Center of Anti-Inflammatory and Immune Medicine, Institute of Clinical Pharmacology, Anhui Medical University; ${ }^{2}$ Department of Neurosurgery, The First Affiliated Hospital of Anhui Medical University, Hefei, Anhui 230032, P.R. China
\end{abstract}

Received May 30, 2019; Accepted April 3, 2020

DOI: 10.3892/ol.2020.11602

\begin{abstract}
Glioma is a type of malignant tumor arising from glial cells of the brain or the spine. Circulation-derived macrophage infiltration is a characteristic of the glioma microenvironment. The polarization status of circulation-derived macrophages in patients with glioma remains unclear. Therefore, the present study aimed to evaluate the polarization status of circulation-derived macrophages in patients with glioma. A total of 40 patients with glioma and 38 healthy volunteers were recruited. The polarization status of macrophage-like cells in the peripheral blood of patients with glioma was evaluated. In addition, the associations between the polarization status of macrophage-like cells and glioma stage or the expression levels of the glioma tumor marker chitinase-3-like protein 1 (also termed YKL-40) were evaluated. The number of macrophage-like cells $\left(\mathrm{CD} 115^{+} \mathrm{CD} 1 \mathrm{c}^{-} \mathrm{CD} 2^{-} \mathrm{CD} 15^{-} \mathrm{CD} 19^{-} \mathrm{CD} 14^{+} \mathrm{CD} 16^{+} \mathrm{CD} 11 \mathrm{~b}^{+}\right)$ was higher in the peripheral blood of patients with glioma compared with that of healthy volunteers. There were fewer M1 macrophage-like cells, and more M2 macrophage-like cells were induced in the peripheral blood of patients with glioma compared with healthy controls. Specifically, the number of M2a/M2b macrophage-like cells increased, whereas that of M2c macrophage-like cells decreased in the peripheral blood of patients with glioma compared with healthy controls. The polarization status of macrophage-like cells in patients with
\end{abstract}

Correspondence to: Professor Jiajie Tu or Professor Wei Wei, Key Laboratory of Anti-Inflammatory and Immune Medicine, Ministry of Education, Anhui Collaborative Innovation Center of Anti-Inflammatory and Immune Medicine, Institute of Clinical Pharmacology, Anhui Medical University, 81 Meishan Road, Hefei, Anhui 230032, P.R. China

E-mail: tujiajie@ahmu.edu.cn

E-mail:wwei@ahmu.edu.cn

*Contributed equally

Key words: macrophage, polarization, glioma, YKL-40 glioma was not significantly associated with glioma stage or with the glioma marker YKL-40. Overall, the results of the present study revealed that the polarization status of macrophage-like cells in the peripheral blood of patients with glioma was abnormal, offering potential novel diagnostic and therapeutic targets, such as different macrophage subsets, for glioma.

\section{Introduction}

Macrophages are a group of immune cells that serve essential roles in both physiological and pathological conditions by being involved in inflammatory and immune responses $(1,2)$. In response to intracellular or extracellular stimulation, the monocyte-macrophage system can transit to two major distinct polarization patterns: The pro-inflammatory M1 type and the anti-inflammatory M2 type, which exhibit contrasting cellular phenotypes and functions (3). M2 type macrophages have a high phenotypic heterogeneity and can be further divided into three subsets: M2a, M2b and M2c (4). The M2a subtype is defined as alternatively activated macrophages, induced by fungal and helminth infections, interleukin (IL)-4 and IL-13; the M2b subtype is defined as type 2 macrophages, induced by immune complexes and lipopolysaccharide; and the M2c subtype is defined as deactivated macrophages, induced by IL-10, transforming growth factor- $\beta$ and glucocorticoids (4).

Glioma is a type of malignant tumor arising from glial cells of the brain or the spine. The heterogeneity of macrophages is high in the glioma microenvironment (5). Gliomas contain two subtypes of macrophages, brain-resident microglia and circulating monocyte-derived macrophages (6). Both of these subtypes have been demonstrated to contribute to glioma progression and maintenance (7). Macrophage transformation from the M1 to the M2 type can promote glioma development $(8,9)$. However, to the best of our knowledge, there are no reports on the polarization status of macrophage-like cells in the peripheral blood of patients with glioma. Chitinase-3-like protein 1, also termed YKL-40, is highly expressed in glioma tissues compared with adjacent normal brain tissues (10). YKL-40 is secreted by tumor cells and tumor-associated macrophages into the blood and has a prognostic value in various types of cancer, such as Hodgkin lymphoma and melanoma $(11,12)$. However, the association between the 
polarization status of macrophage-like cells in the peripheral blood and tumor stage or YKL-40 expression in patients with glioma remains unclear.

The development of diagnostic and therapeutic strategies for glioma has greatly improved during the past decades, but glioma remains one of the most malignant tumors worldwide (3-8 cases/100,000 individuals) (13). Based on the advances in cancer immunotherapy and the role of macrophages in glioma development, novel immunological markers and potential therapeutic targets of macrophages should be considered in glioma research. Therefore, the present study aimed to investigate the polarization status of macrophage-like cells in the peripheral blood of patients with glioma and to evaluate the associations among macrophage-like cell polarization patterns, glioma severity and the glioma marker YKL-40 in the peripheral blood of patients with glioma.

\section{Materials and methods}

Patients. Blood samples were obtained from 40 patients with glioma and 38 healthy controls (all Chinese) at The First Affiliated Hospital of Anhui Medical University (Hefei, China). Glioma tissues and adjacent normal tissues were obtained from 40 patients with glioma (all Chinese) upon excision surgery at the Department of Neurosurgery of The First Affiliated Hospital of Anhui Medical University. The patient characteristics are summarized in Table I. Patients with glioma (average age, 52.7 years; age range, 8-82 years) and healthy controls (average age, 39.7 years; age range, 23-62 years) were recruited and their blood and tumor samples were collected between May 2017 and August 2018. Specifically, adjacent normal tissues were excised from non-functional tissues within $2 \mathrm{~cm}$ from the tumor tissues. Blood samples and tumor tissues were collected from the same 40 patients. The staging of glioma was based on the 2016 World Health Organization Classification of Tumors of the Central Nervous System (14). The present study was approved by the Ethics Committee of Anhui Medical University. Informed consent was provided by all participants or their guardians. The histological types of the included patients were limited to glioma (including glioblastoma, mesoglioma, ganglioglioma, astrocytic glioma and spongiocytoma). Patients with incomplete information (age, sex, histological type, stage, recurrence or received chemotherapy) were excluded.

Blood samples. Peripheral venous blood $(4 \mathrm{ml})$ was collected from each participant. Peripheral blood mononuclear cells were isolated using a PBMC isolation kit (cat. no. DKW-KLSH-0100; DAKEWE, Inc.) for use in subsequent flow cytometry analysis. For patients with glioma, blood samples were collected before the surgical procedure.

Flow cytometry. A flow cytometric gating strategy $\left(\mathrm{CD} 115^{+} \mathrm{C}\right.$ D1c ${ }^{-} \mathrm{CD} 2{ }^{-\mathrm{CD}} 15^{-\mathrm{CD}} 19^{-} \mathrm{CD} 14^{+} \mathrm{CD} 16^{+} \mathrm{CD} 11 \mathrm{~b}^{+}$) was used to stain macrophage-like cells in the peripheral blood of patients with glioma and healthy controls. CD115 was used to select monocyte-lineage cells, dendritic cells were excluded using CD1c, $\mathrm{B}$ cells were excluded using CD19, $\mathrm{T}$ and NK cells were excluded using CD2, and granulocytes were excluded using CD15. Macrophage-like cells were selected using the macrophage antibodies CD14, CD16 and CD11b (all Miltenyi Biotec, Inc.).

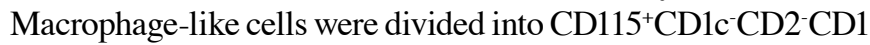

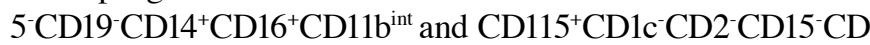
${ }^{19} \mathrm{CD} 14^{+} \mathrm{CD} 16^{+} \mathrm{CD} 11 \mathrm{~b}^{\mathrm{hi}}$ subsets for subsequent analyses; $\mathrm{CD} 11 \mathrm{~b}^{\mathrm{int}}$ (P6, Vioblue $\left.1 \times 10^{2}-1 \times 10^{4}\right)$ and $\mathrm{CD} 11 \mathrm{~b}^{\mathrm{hi}}\left(\mathrm{P} 7\right.$, Vioblue $\left.1 \times 10^{4}-1 \times 10^{6}\right)$ were decided based on the fluorescence-activated cell sorting (FACS) strategy shown in Fig. S1. In CD11b macrophage-like cells, $\mathrm{CCR} 7^{+} \mathrm{CD} 86^{+}$was used to mark the $\mathrm{M} 1$ type, while CCR7-CXCR1 ${ }^{+}, \mathrm{CCR}^{-}{ }^{-\mathrm{CD}} 86^{+}$and CCR7-CCR2 ${ }^{+}$were used to identify the M2a, M2b and M2c macrophage-like cells, respectively (15). The cells were analyzed using the BD FACSAria II flow cytometer and the BD FACSDiva software v8.0.2 (both BD Biosciences). Glioma tissues and adjacent normal tissues were incubated with the following fluorescein isothiocyanate (FITC)-, phycoerythrin (PE)-, allophycocyanin (APC), PE-Cy7-, APC-Cy7- or Vioblue-conjugated monoclonal antibodies at $4^{\circ} \mathrm{C}$ for $1 \mathrm{~h}$ : Rat anti-human CD115-PE ( $2 \mu \mathrm{g} / \mathrm{test}$; cat. no. 347303) was from BioLegend, Inc., whereas mouse anti-human CD1c-FITC (5 $\mu \mathrm{g} /$ test; cat. no. 130-113-863), CD2-FITC $(5 \mu \mathrm{g} /$ test; cat. no. 130-098-685), CD15-FITC (5 $\mu \mathrm{g} /$ test; cat. no. 130-114-010), CD19-FITC (5 $\mu \mathrm{g} / \mathrm{test}$; cat. no. 130-114-171), CD14-APC ( $2 \mu \mathrm{g} /$ test; cat. no. 130-110-578), CD16-APC-Cy7 ( $2 \mu \mathrm{g} /$ test; cat. no. 130-113-952), CD11b-Vioblue ( $2 \mu \mathrm{g} /$ test; cat. no. 130-110-616), CD86-APC ( $\mu \mathrm{g} / \mathrm{test}$; cat. no. 130-114-095), CCR2-PE (2 $\mu \mathrm{g} /$ test; cat. no. 130-109-654), CCR7-FITC (5 $\mu \mathrm{g} /$ test; cat. no. 130-117-700) and CXCR1-PE-Cy7 (2 $\mu \mathrm{g} / \mathrm{test}$; cat. no. 130-115-950) were from Miltenyi Biotec, Inc.

Immunohistochemistry (IHC) and hematoxylin and eosin $(H \& E)$ staining. IHC and H\&E staining were performed as previously described (16). Briefly, paraffin-embedded tissue slides obtained from glioma and adjacent tissues were deparaffinized in xylene twice for 5 min each at room temperature. Subsequently, slides were transferred in $100 \%$ alcohol twice for 3 min each, and then rehydrated in a descending alcohol series (95, 70 and 50\%) for 3 min each. The EDTA antigen retrieval buffer (Beijing Solarbio Science \& Technology Co., Ltd.) was heated until boiling and added to the sections; this was repeated two more times every 5-10 min. The slides were left to cool down at room temperature and washed with double-distilled $\mathrm{H}_{2} \mathrm{O}$. Subsequently, the slides were blocked in 5\% goat serum (Biological Industries) for $60 \mathrm{~min}$ at room temperature, and subsequently incubated with primary monoclonal rabbit anti-human anti-inducible nitric oxide synthase (iNOS) (1:100; cat. no. sc-651) and polyclonal sheep anti-human anti-CD206 (1:50; cat. no. sc-34577; both from Santa Cruz Biotechnology, Inc.) antibodies at $4^{\circ} \mathrm{C}$ for $12 \mathrm{~h}$. For H\&E staining, sections were deparaffinized in xylene, re-hydrated in absolute alcohol and washed briefly with distilled water. Subsequently, they were stained with Harris hematoxylin solution and washed with running tap water, differentiated in $1 \%$ acid alcohol and washed with running tap water, blued in $0.2 \%$ saturated lithium carbonate solution and washed with running tap water, rinsed in $95 \%$ alcohol and counterstained with eosin-phloxine solution, dehydrated in alcohol and cleared in xylene, and finally mounted with mounting medium (xylene-based). ImageJ software (version d 1.47; National Institutes of Health) was used for quantification of IHC.

Calculation of M1 and M2 macrophage infiltration. In areas of iNOS ${ }^{+}$and $\mathrm{CD}_{206}{ }^{+}$macrophage infiltration, individual 
Table I. Summary of patient characteristics.

\begin{tabular}{|c|c|c|}
\hline Characteristic & Patient no. & Percentage \\
\hline \multicolumn{3}{|l|}{ Age, years } \\
\hline$<50$ & 16 & 40.0 \\
\hline$\geq 50$ & 24 & 60.0 \\
\hline \multicolumn{3}{|l|}{ Sex } \\
\hline Male & 22 & 55.0 \\
\hline Female & 18 & 45.0 \\
\hline \multicolumn{3}{|c|}{ Histopathological type } \\
\hline Glioblastoma & 23 & 57.5 \\
\hline Mesoglioma & 5 & 12.5 \\
\hline Ganglioglioma & 3 & 7.5 \\
\hline Astrocytic glioma & 5 & 12.5 \\
\hline Spongiocytoma & 4 & 10.0 \\
\hline \multicolumn{3}{|l|}{ Stage $^{\mathrm{a}}$} \\
\hline 0 & 0 & 0.0 \\
\hline I & 2 & 5.0 \\
\hline II & 11 & 27.5 \\
\hline III & 7 & 17.5 \\
\hline IV & 20 & 50.0 \\
\hline \multicolumn{3}{|l|}{ Recurrence } \\
\hline Yes & 9 & 22.5 \\
\hline No & 31 & 77.5 \\
\hline \multicolumn{3}{|c|}{ Received chemotherapy } \\
\hline Yes & 5 & 12.5 \\
\hline No & 35 & 87.5 \\
\hline
\end{tabular}

astaging according to the 2016 World Health Organization Classification of Tumors of the Central Nervous System.

macrophage infiltration was measured on a x200 magnification field using an Olympus BX53 light microscope. An iNOS ${ }^{+}$and CD206 ${ }^{+}$macrophage cluster was counted as a single infiltrated macrophage. The ratio of M1 and M2 infiltrated macrophages was calculated as the absolute number of iNOS $^{+}$and CD206 ${ }^{+}$macrophages/total cells per $\mathrm{x} 200$ magnification field $(n=8)$.

Statistical analysis. GraphPad Prism v7 (GraphPad Software, Inc.) was used for statistical analysis. Variances were first assessed by Bartlett's test. Subsequently, significant differences between two groups were analyzed using a two-tailed unpaired Student's t-test when equal variances were assumed, or a Welch's t-test for unequal variances. $\mathrm{P}<0.05$ was considered to indicate a statistically significant difference.

\section{Results}

Detection of $C D 11 b^{i n t}$ and $C D 11 b^{\text {hi }}$ peripheral blood macrophage-like cells in patients with glioma. A total of 40 patients with glioma and 38 healthy controls were recruited in the present study. The clinical and pathological features of the patients are listed in Table I. Macrophage-like cells in the peripheral blood were detected by flow cytometry with CD115

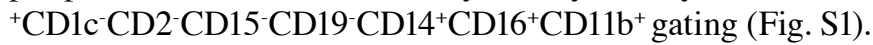

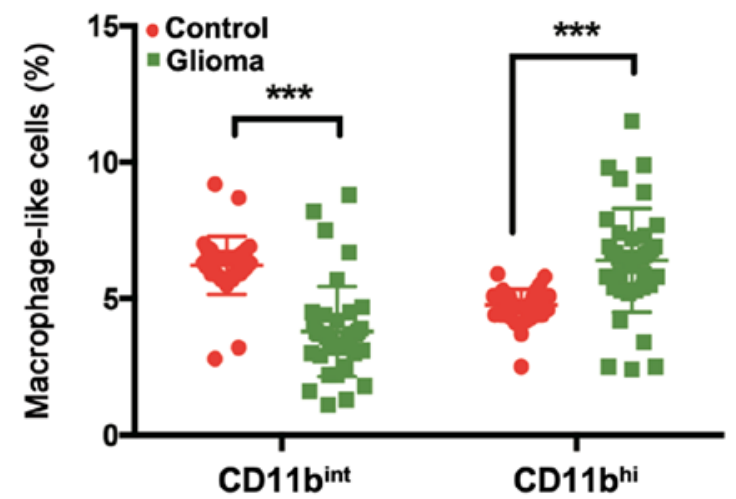

Figure 1. Comparison of percentages of CD11b $b^{\text {int }}$ and $C D 11 b^{\text {hi }}$ peripheral blood macrophage-like cells in patients with glioma and healthy controls. ${ }^{* * * *} \mathrm{P}<0.001$. int, intermediate; hi, high.

There were two distinct populations of macrophage-like cells: CD $115^{+} \mathrm{CD} 1 \mathrm{c}^{-\mathrm{CD}} 2^{-\mathrm{CD}} 15^{-\mathrm{CD}} 19^{-\mathrm{CD}} 14^{+} \mathrm{CD} 16^{+} \mathrm{CD} 11 \mathrm{~b}^{\mathrm{int}}$ and CD $115^{+} \mathrm{CD} 1 \mathrm{c}^{-} \mathrm{CD} 2^{-\mathrm{CD}} 15^{-\mathrm{CD}} 19^{-\mathrm{CD}} 14^{+} \mathrm{CD} 16^{+} \mathrm{CD} 11 \mathrm{~b}^{\mathrm{hi}}$ cells. The number and percentage of $\mathrm{CD} 115^{+} \mathrm{CD} 1 \mathrm{c}^{-} \mathrm{CD} 2^{-} \mathrm{CD} 15^{-} \mathrm{CD}$ $19^{-} \mathrm{CD} 14^{+} \mathrm{CD} 16^{+} \mathrm{CD} 11 b^{\text {int }}$ cells among peripheral blood monocytes from patients with glioma were significantly decreased compared with those from healthy controls (Fig. 1). By contrast, an increased percentage of $\mathrm{CD} 115^{+} \mathrm{CD} 1 \mathrm{c}^{-} \mathrm{CD} 2^{-} \mathrm{CD} 15^{-} \mathrm{CD} 19^{-} \mathrm{CD}$ $14^{+} \mathrm{CD} 16^{+} \mathrm{CD} 11 \mathrm{~b}^{\mathrm{hi}}$ cells was observed in the peripheral blood of patients with glioma compared with those in the blood of healthy controls (Fig. 1).

Polarization patterns of $C D 11 b^{\text {int }}$ and $C D 11 b^{\text {hi }}$ peripheral blood macrophage-like cells in patients with glioma. In CD11 $\mathrm{b}^{+}$ (including both $\mathrm{CD} 11 \mathrm{~b}^{\mathrm{int}}$ and $\mathrm{CD} 11 \mathrm{~b}^{\mathrm{hi}}$ ) macrophage-like cells, the percentage of $\mathrm{M} 1$ type cells $\left(\mathrm{CCR} 7^{+} \mathrm{CD} 86^{+}\right)$was significantly lower in patients with glioma compared with that in healthy controls (Figs. 2B and 3C). However, there were almost no M2 type cells (CCR7) in the CD11b ${ }^{\text {int }}$ population (Fig. 2A). In the $C D 11 b^{\text {hi }}$ cell population, the percentage of total M2 type cells was higher in patients with glioma compared with that in healthy controls (Fig. 3A, B and D). Specifically, M2a (CCR7-CXCR1 ${ }^{+}$) and $\mathrm{M} 2 \mathrm{~b}\left(\mathrm{CCR} 7^{-} \mathrm{CD} 86^{+}\right)$type cells were upregulated, whereas $\mathrm{M} 2 \mathrm{c}$ type cells $\left(\mathrm{CCR} 7^{-} \mathrm{CCR} 2^{+}\right)$were downregulated in the peripheral blood of patients with glioma compared with those in the blood of healthy controls (Fig. 3E-G).

Polarization patterns of macrophages in glioma tissues. To validate this polarization pattern, glioma and adjacent normal brain tissues were used for detecting the well-established M1 (iNOS) and M2 (CD206) markers (Fig. 4). The typical morphology of glioma and adjacent normal brain tissues is shown in Fig. 4A. Quantification of the IHC results revealed that the percentage of the M2 marker $\mathrm{CD} 206^{+}$cells was higher than the percentage of the M1 marker iNOS ${ }^{+}$cells in glioma tumor tissues (Fig. 4B and C). Additionally, FACS analysis validated that macrophages expressed higher levels of the M2 marker CD206 than the M1 marker iNOS in glioma tissues (Fig. 4D and E). The present results suggested that patients with glioma exhibited a distinct pattern of macrophage polarization in the peripheral blood, namely a lower percentage of M1 type cells and a higher percentage of M2 type cells 
A

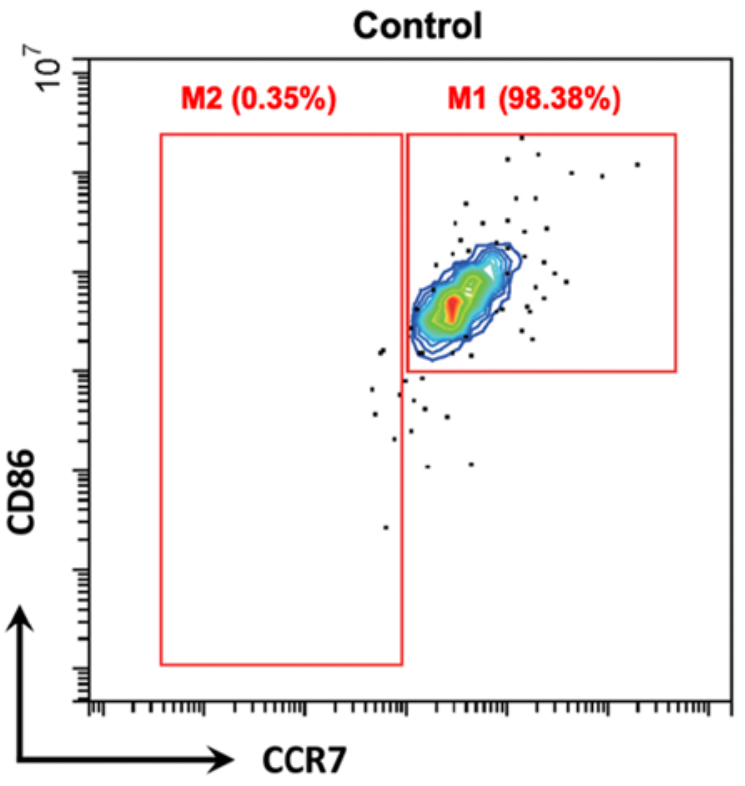

Glioma

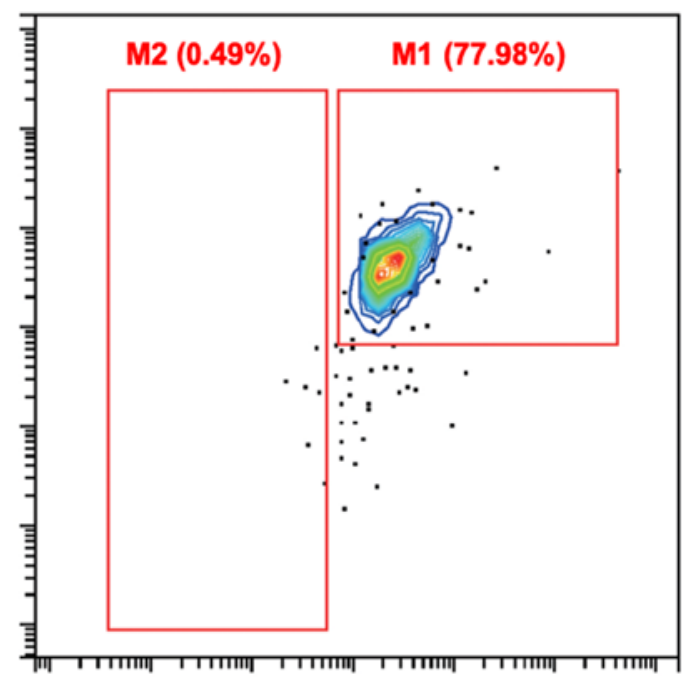

B

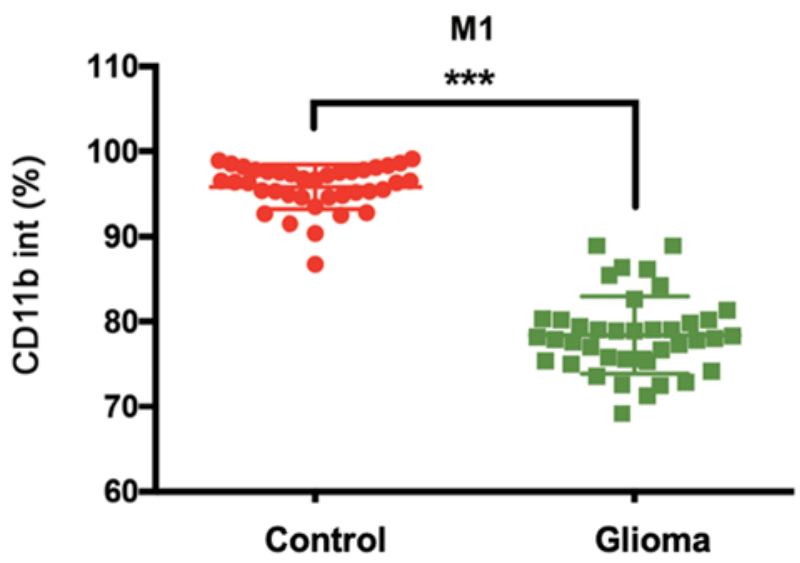

Figure 2. Comparison of CD11b ${ }^{\text {int }}$ peripheral blood macrophage-like cells in patients with glioma and healthy controls. (A) Fluorescence-activated cell sorting results of M1 and M2 patterns of CD11b ${ }^{\text {int }}$ peripheral blood macrophage-like cells in patients with glioma and controls. (B) Quantification of M1 patterns of $\mathrm{CD}_{1} \mathrm{~b}^{\text {int }}$ peripheral blood macrophage-like cells in patients with glioma and healthy controls ${ }^{* * *} \mathrm{P}<0.001$. int, intermediate.

(especially M2a and M2b type cells) compared with healthy controls.

Subsequently, the patterns of macrophage polarization in patients with different stages of glioma were evaluated. The percentages of macrophage-like cells in the peripheral blood were not significantly different between patients with advanced glioma (stages III and IV) and those with early glioma (stages I and II) (Fig. S2). Since previous studies have suggested that serum YKL-40 is a candidate marker for glioma (17-19), the present study investigated the association between the patterns of macrophage polarization and serum YKL-40 status in patients with glioma. However, the percentages of macrophage-like cells were not significantly different between patients with low YKL-40 expression and those with high YKL-40 expression (Fig. S3).

\section{Discussion}

Currently, a large proportion of the mortality of patients with glioma is caused by tumor recurrence, indicating an urgent requirement for improved diagnosis and therapy $(20,21)$.
However, no specific diagnostic clinical marker for glioma has yet been identified. For example, YKL-40 is a potential specific marker of prognosis in high-grade glioma, but not in low-grade glioma $(17,22,23)$. A previous study has demonstrated that tumor-associated macrophages (TAMs) are infiltrated in the glioma microenvironment and can facilitate survival, migration and neovascularization of glioma; therefore, TAMs are considered as a potential therapeutic target for glioma treatment (7). Although a large proportion of TAMs in glioma come from the peripheral circulation, to the best of our knowledge, the polarization of macrophage-like cells in the peripheral blood of patients with glioma, or the association between macrophage-like cells from the peripheral blood and TAMs infiltrated in the glioma environment, have not yet been systemically evaluated. In the present study, an abnormal polarization pattern of macrophage-like cells was observed in the peripheral blood of patients with glioma. The percentages of $\mathrm{CD} 115^{+} \mathrm{CD} 1 \mathrm{c}^{-} \mathrm{CD} 22^{-} \mathrm{CD} 15^{-\mathrm{CD}} 19^{-} \mathrm{CD} 14^{+} \mathrm{CD} 16^{+} \mathrm{CD} 11 \mathrm{~b}^{\mathrm{int}}$ and $\mathrm{CD} 115^{+} \mathrm{CD} 1 \mathrm{c}^{-\mathrm{CD}} 2^{-\mathrm{CD}} 15^{-\mathrm{CD}} 19^{-\mathrm{CD}} 14^{+} \mathrm{CD} 16^{+} \mathrm{CD} 1$ $1 b^{\text {hi }}$ macrophage-like cells were significantly altered in 

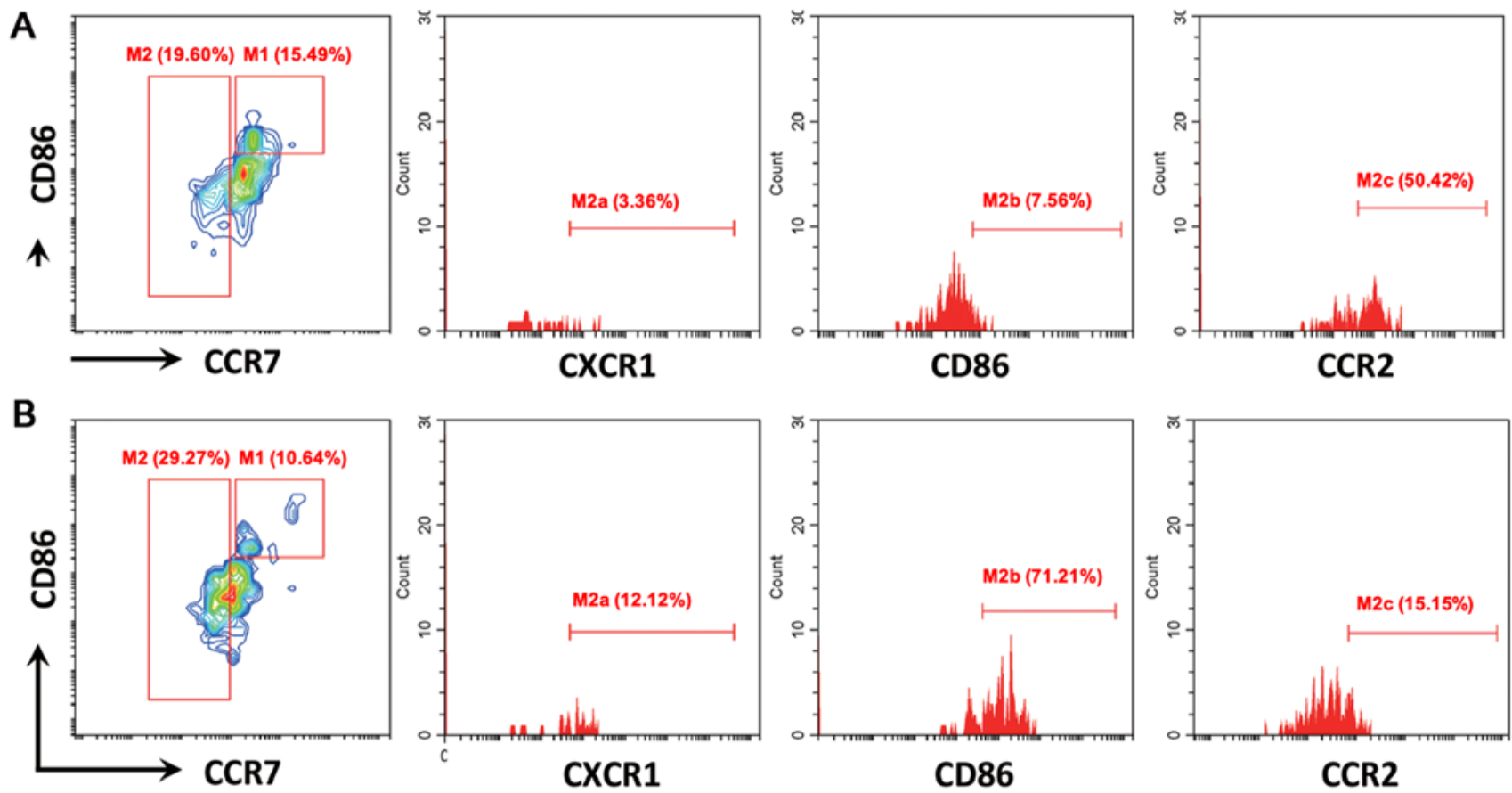

C

D Total M2
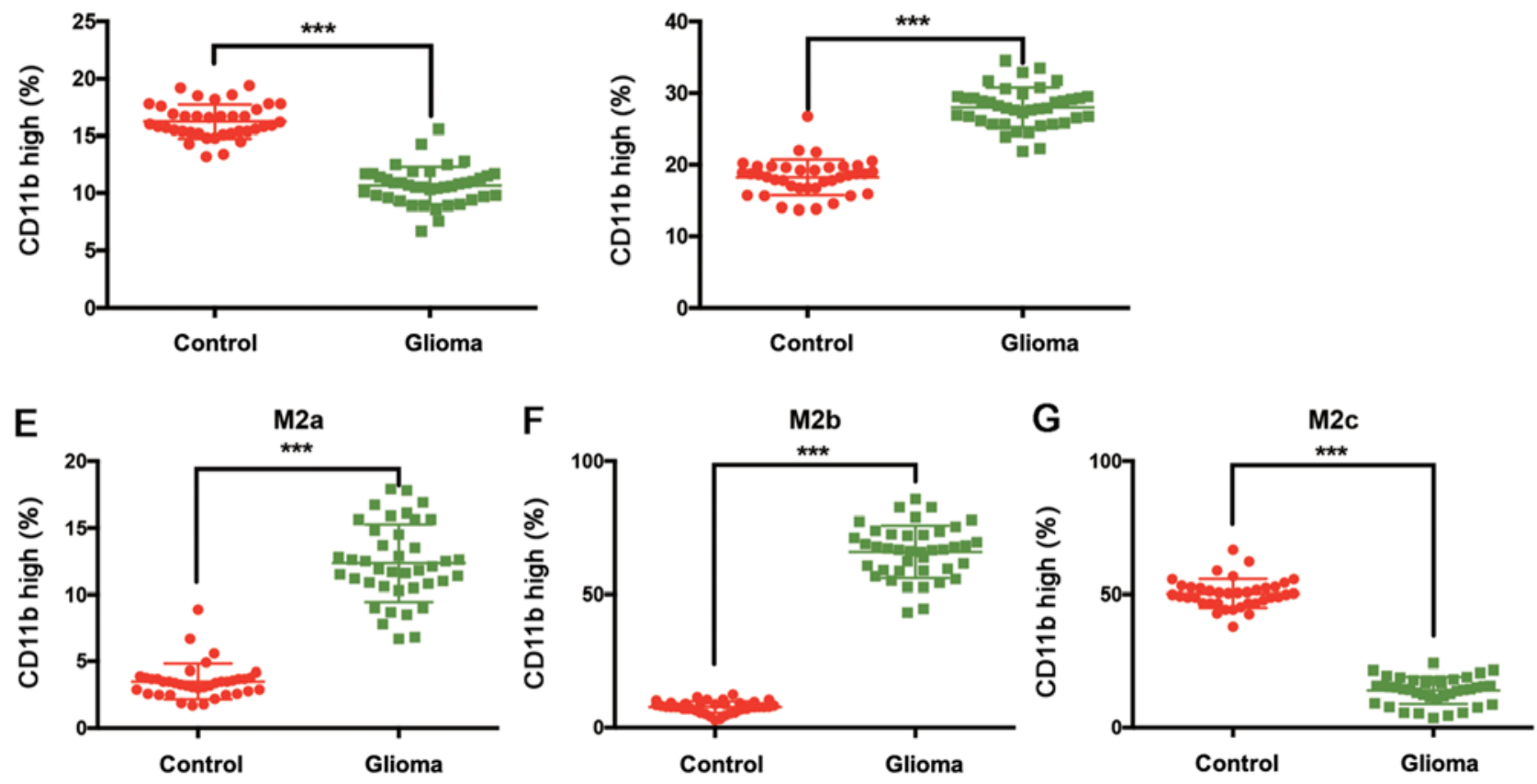

Figure 3. Comparison of polarization patterns of CD11 b ${ }^{\text {hi }}$ peripheral blood macrophage-like cells in patients with glioma and healthy controls Fluorescence-activated cell sorting results of M1 and M2 patterns of CD11b ${ }^{\text {hi }}$ peripheral blood macrophage-like cells in (A) controls and (B) patients with glioma. Quantification of (C) M1 and (D) M2 patterns of CD11b ${ }^{\text {hi }}$ peripheral blood macrophage-like cells in patients with glioma and healthy controls. Comparison of (E) M2a, (F) M2b and (G) M2c type cells among CD1 $1 b^{\text {hi }}$ peripheral blood macrophage-like cells in patients with glioma and healthy controls.

${ }^{* * * *} \mathrm{P}<0.001$. hi, high.

patients with glioma compared with those in healthy controls, suggesting that these cells may be potential markers for the diagnosis and monitoring of glioma.

Classical M1 polarization of the monocyte-macrophage system has demonstrated anticancer activity in different types of cancer (24,25). Alternatively activated M2 macrophages generally act as tumor promoters and include three subtypes: M2a, M2b and M2c (26). Different M2 subtypes serve different roles in tumorigenesis (27). The present study demonstrated that the numbers of M1 macrophages in the peripheral blood of patients with glioma were decreased compared with those in healthy controls, indicating poor antitumor capacity of circulating macrophage-like cells in patients with glioma. By contrast, the numbers of M2 macrophages were increased in patients with glioma compared with those in healthy controls, indicating that more M2 macrophage-like cells may be recruited in glioma and may infiltrate the glioma microenvironment in the brain. 

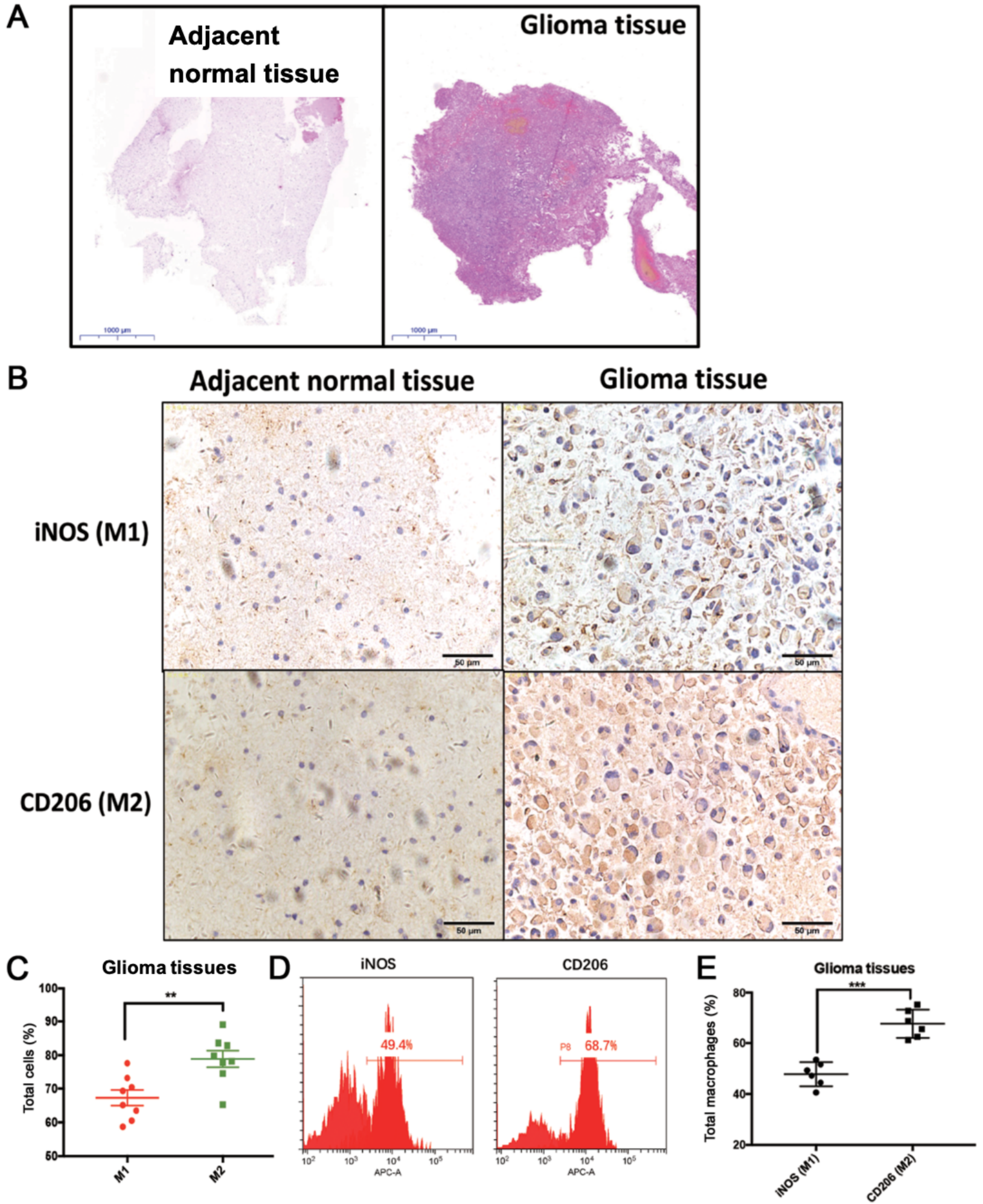

Figure 4. Polarization patterns of macrophages in glioma tissues. (A) Hematoxylin and eosin staining of glioma and adjacent normal tissues. (B) Macrophage iNOS (M1) and CD206 (M2) marker expression in glioma and adjacent normal tissues assessed by immunohistochemistry. (C) Quantification of iNOS $^{+}$and $\mathrm{CD}_{206}{ }^{+}$cells in glioma tissues. (D and E) Macrophage M1 and M2 marker expression in glioma tumor tissues determined by fluorescence-activated cell sorting. ${ }^{* *} \mathrm{P}<0.01 ;{ }^{* * *} \mathrm{P}<0.001$. iNOS, inducible nitric oxide synthase.

Finally, the associations of macrophage-like cell polarization patterns with glioma stage or with the glioma marker YKL-40 were evaluated in the present study. Of note, no significant association was identified between different tumor stages and the polarization patterns of circulating macrophage-like cells. YKL-40 is abundantly expressed in glioma, and various studies have reported the oncogenic role of this gene (17-19). Additionally, it has been demonstrated that YKL-40 is secreted by both tumor cells and tumor-associated macrophages $(28,29)$. However, the present study revealed that neither the percentage nor the polarization status of macrophages in the $\mathrm{CD} 115^{+} \mathrm{CD}$ $1 c^{-} \mathrm{CD} 2{ }^{-\mathrm{CD}} 15^{-\mathrm{CD}} 19^{-\mathrm{CD}} 14^{+} \mathrm{CD} 16^{+} \mathrm{CD} 11 \mathrm{~b}^{\mathrm{int}}$ and $\mathrm{CD} 115^{+} \mathrm{CD} 1 \mathrm{c}$ 
$\mathrm{CD} 2{ }^{-} \mathrm{CD} 15^{-} \mathrm{CD} 19^{-} \mathrm{CD} 14^{+} \mathrm{CD} 16^{+} \mathrm{CD} 11 \mathrm{~b}^{\text {hi }}$ subsets was different between patients with high YKL-40 expression and those with low YKL-40 expression. The present results should be further confirmed by evaluating the association between infiltrated macrophages and YKL-40 expression within the tumor microenvironment. In addition, other monocyte-derived cells, such as dendritic cells (DCs), are recruited to the tumor tissue (30) and express YKL-40 (31). Therefore, the function of YKL-40-secreting DCs should not be neglected in glioma.

Efficient clinical biomarkers and targets are required for timely and effective glioma diagnosis and therapy. Although the present study only recruited 40 patients with glioma, the aberrant polarization patterns of macrophage-like cells in the peripheral blood may represent potential serum biomarkers for glioma diagnosis and monitoring. Specifically, the upregulation of M2a and $\mathrm{M} 2 \mathrm{~b}$ cells, and the downregulation of M2c cells in the peripheral blood of patients with glioma require further validation in glioma tissues using a large cohort of clinical samples. Additionally, future studies should evaluate the specific function of circulating macrophage-like cells in the brain glioma tissue. For example, co-culturing primary infiltrated macrophages and glioma cells could illustrate the communication between these two types of cells. Finally, the effects of immunoregulatory drugs and agents on the polarization status of macrophages should be examined in glioma, such as the interaction between the programmed death 1(PD-1)/programmed death ligand 1 (PD-L1) pathway and the polarization of macrophage-like cells in the peripheral blood of patients with glioma. The polarization status of macrophages may be a potential marker for the diagnosis and monitoring of patients who received PD-1/PD-L1 checkpoint therapy (32). Overall, the present study revealed the polarization status of macrophage-like cells in the peripheral blood of patients with glioma. The potential prognostic application of the abnormal polarization patterns of circulating macrophage-like cells in glioma should be further evaluated in future studies.

\section{Acknowledgements}

Not applicable.

\section{Funding}

The present study was supported by the National Natural Science Foundation of China (grant nos. 81673444 and 31900616), the Natural Science Foundation of Anhui Province for young scholars (grant no. 1908085QH379) and Grants for Scientific Research of BSKY from Anhui Medical University (grant no. 4501041101).

\section{Availability of data and materials}

The datasets used and/or analyzed during the present study are available from the corresponding author on reasonable request.

\section{Authors' contributions}

YG and WH performed the experiments. PZ, DH, YF, JT and WW interpreted the results and drafted the manuscript. YG revised the manuscript. All authors read and approved the final manuscript.

\section{Ethics approval and consent to participate}

The present study was approved by the Ethics Committee of Anhui Medical University, and informed consent was obtained from all participants or their guardians.

\section{Patient consent for publication}

Not applicable.

\section{Competing interests}

The authors declare that they have no competing interests.

\section{References}

1. Ariel A, Maridonneau-Parini I, Rovere-Querini P, Levine JS and Mühl H: Macrophages in inflammation and its resolution. Front Immunol 3: 2-3, 2012.

2. Liu YC, Zou XB, Chai YF and Yao YM: Macrophage polarization in inflammatory diseases. Int J Biol Sci 10: 520-529, 2014.

3. Murray PJ: Macrophage polarization. Annu Rev Physiol 79: 541-566, 2017.

4. Roszer T: Understanding the mysterious M2 macrophage through activation markers and effector mechanisms. Mediators Inflamm 2015: 16-18, 2015.

5. Kurowska-Stolarska M and Alivernini S: Synovial tissue macrophages: Friend or foe? RMD Open 3: e000527, 2017.

6. Müller S, Kohanbash G,Liu SJ, Alvarado B, Carrera D, Bhaduri A, Watchmaker PB, Yagnik G, Di Lullo E, Malatesta M, et al: Single-cell profiling of human gliomas reveals macrophage ontogeny as a basis for regional differences in macrophage activation in the tumor microenvironment. Genome Biol 18: 234 , 2017.

7. Hambardzumyan D, Gutmann DH and Kettenmann H: The role of microglia and macrophages in glioma maintenance and progression. Nat Neurosci 19: 20-27, 2015.

8. Rhee I: Diverse macrophages polarization in tumor microenvironment. Arch Pharm Res 39: 1588-1596, 2016.

9. Yuan R, Li S, Geng H, Wang X, Guan Q, Li X, Ren C and Yuan X: Reversing the polarization of tumor-associated macrophages inhibits tumor metastasis. Int Immunopharmacol 49: 30-37, 2017.

10. Tanwar MK, Gilbert MR and Holland EC: Gene expression microarray analysis reveals YKL-40 to be a potential serum marker for malignant character in human glioma. Cancer Res 62: 4364-4368, 2002 .

11. Biggar RJ, Johansen JS, Smedby KE, Rostgaard K, Chang ET, Adami HO, Glimelius B, Molin D, Hamilton-Dutoit S, Melbye $\mathrm{M}$ and Hjalgrim H: Serum YKL-40 and interleukin 6 levels in Hodgkin lymphoma. Clin Cancer Res 14: 6974-6978, 2008.

12. Schmidt H, Johansen JS, Gehl J, Geertsen PF, Fode K and von der Maase H: Elevated serum level of YKL-40 is an independent prognostic factor for poor survival in patients with metastatic melanoma. Cancer 106: 1130-1139, 2006.

13. Tamura Y, Kato H, Oh A, Kisanuki K, Tsuchiya S, Terashima G and Shimasaki Y: Glioblastoma and other malignant gliomas a clinical review. JAMA 310: 1842-1850, 2013.

14. Louis DN, Perry A, Reifenberger G, von Deimling A, Figarella-Branger D, Cavenee WK, Ohgaki H, Wiestler OD, Kleihues P and Ellison DW: The 2016 world health organization classification of tumors of the central nervous system: A summary. Acta Neuropathol 131: 803-820, 2016.

15. Hung CH, Chen FM, Lin YC, Tsai ML, Wang SL, Chen YC, Chen YT and Hou MF: Altered monocyte differentiation and macrophage polarization patterns in patients with breast cancer. BMC Cancer 18: 366, 2018.

16. Tu J, Cheung HH, Lu G, Chen Z and Chan WY: MicroRNA-10a promotes granulosa cells tumor development viaPTEN-AKT/Wnt regulatory axis. Cell Death Dis 9: 1076, 2018.

17. Nutt CL, Betensky RA, Brower MA, Batchelor TT, Louis DN and Stemmer-Rachamimov AO: YKL-40 is a differential diagnostic marker for histologic subtypes of high-grade gliomas. Clin Cancer Res 11: 2258-2264, 2005. 
18. Hormigo A, Gu B, Karimi S, Riedel E, Panageas KS, Edgar MA, Tanwar MK, Rao JS, Fleisher M, DeAngelis LM and Holland EC: YKL-40 and matrix metalloproteinase-9 as potential serum biomarkers for patients with high-grade gliomas. Clin Cancer Res 12: 5698-5704, 2006.

19. Ku BM, Lee YK, Ryu J, Jeong JY, Choi J, Eun KM, Shin HY, Kim DG, Hwang EM, Yoo JC, et al: CHI3L1 (YKL-40) is expressed in human gliomas and regulates the invasion, growth and survival of glioma cells. Int J Cancer 128: 1316-1326, 2011.

20. Ludwig K and Kornblum HI: Molecular markers in glioma. J Neurooncol 134: 505-512, 2017.

21. Agnihotri S, Burrell KE, Wolf A, Jalali S, Hawkins C, Rutka JT and Zadeh G: Glioblastoma, a brief review of history, molecular genetics, animal models and novel therapeutic strategies. Arch Immunol Ther Exp (Warsz) 61: 25-41, 2013.

22. Iwamoto FM, Hottinger AF, Karimi S, Riedel E, Dantis J, Jahdi M, Panageas KS, Lassman AB, Abrey LE, Fleisher M, et al: Serum YKL-40 is a marker of prognosis and disease status in high-grade gliomas. Neuro Oncol 13: 1244-1251, 2011.

23. Zhao YH, Pan ZY, Wang ZF, Ma C, Weng H and Li ZQ: YKL-40 in high-grade glioma: Prognostic value of protein versus mRNA expression. Glioma 1: 104-110, 2018.

24. Ostuni R, Kratochvill F, Murray PJ and Natoli G: Macrophages and cancer: From mechanisms to therapeutic implications. Trends Immunol 36: 229-239, 2015.
25. Cheng $\mathrm{H}$, Wang Z, Fu L and Xu T: Macrophage polarization in the development and progression of ovarian cancers: An overview. Front Oncol 9: 421, 2019.

26. Mantovani A, Sica A, Sozzani S, Allavena P, Vecchi A and Locati M: The chemokine system in diverse forms of macrophage activation and polarization. Trends Immunol 25: 677-686, 2004.

27. Martinez FO, Sica A, Mantovani A and Locati M: Macrophage activation and polarization. Bioscience 13: 453-461, 2008.

28. Shao R: YKL-40 acts as an angiogenic factor to promote tumor angiogenesis. Front Physiol 4: 122, 2013.

29. Horbinski C, Wang G and Wiley CA: YKL-40 is directly produced by tumor cells and is inversely linked to EGFR in glioblastomas. Int J Clin Exp Pathol 3: 226-237, 2010.

30. RicardC, Tchoghandjian A,Luche H, GrenotP,Figarella-BrangerD, Rougon G, Malissen M and Debarbieux F: Phenotypic dynamics of microglial and monocyte-derived cells in glioblastoma-bearing mice. Sci Rep 6: 26381, 2016

31. Di Rosa M, Tibullo D, Saccone S, Distefano G, Basile MS, Di Raimondo F and Malaguarnera L: CHI3L1 nuclear localization in monocyte derived dendritic cells. Immunobiology 221: 347-356, 2016.

32. Zhang Y, Du W, Chen Z and Xiang C: Upregulation of PD-L1 by SPP1 mediates macrophage polarization and facilitates immune escape in lung adenocarcinoma. Exp Cell Res 359: 449-457, 2017. 